EESTI NSV TEADUSTE AKADEEMIA TOIMETISED. XV KÖIDE FUUSIKA-MATEMAATIKA- JA TEHNIKATEADUSTE SEERIA, 1966, NR. 1

ИЗВЕСТИЯ АКАДЕМИИ НАУК ЭСТОНСКОН ССР. ТОМ ХV СЕРИЯ ФИЗИКО-МАТЕМАТИЧЕСКИХ И ТЕХНИЧЕСКИХ НАУК. 1966, № 1

\title{
ВЛИЯНИЕ ФОРМЫ НАЧАЛЬНОГО ПРОФИЛЯ СКОРОСТИ НА РАЗВИТИЕ ПЛОСКОПАРАЛЛЕЛЬНОЙ ЗАТОПЛЕННОЙ ТУРБУЛЕНТНОЙ СТРУИ
}

Замена мгновенных величин в уравнениях пограничного слоя суммой осредненных и пульсационных приводит к увеличению числа неизвестных и требует дополпительных уравнений. Для большинства случаев эти уравнения могут быть получены только на базе полуэмпирических теорий. Поэтому при решении всех без исключения задач свободной турбулентности для совмещения результатов расчета с экспериментальными данными приходится пользоваться экспериментальными функциями или постояннымн. В идеальном случае эти константы должны включать только пензвестные связи между пульсационными величинами. Однако, как правило, опи включают и все неточностн и несовершенства расчетной схемы, не учитывающей начальных условий истечения. В связи с этим многие экспериментальные данные теряют практическую ценность, если не приведены в соответствующей форме начальные условия.

В частности, очень важно уметь учитывать влияние формы начального профиля на развитие одиночной затопленной струи, что позволяет делать широко распространенный метод Г. Абрамовича ['].

Ниже предлагается метод для расчета плоскопараллельной струи с параболическим распределением начальных импульсов, основанный в отличие от ['] на представлении 06 асимптотическом характере пограничного слоя. На этом представлении базируется решение многих струевых задач, для которых данная методика может оказаться полезной.

В основу расчета положена предложенная Л. Вулисом $\left[{ }^{2}\right]$ линеаризация уравнений турбулентного пограничного слоя путем деформации продольной координаты $x$. При этом уравнение движения превращается в линейное уравнение типа теплопроводности в деформированном пространстве

$$
\frac{\partial \varrho u^{2}}{\partial \tau}=\frac{\partial^{2} \varrho u^{2}}{\partial y^{2}}
$$

где $u$ - продольная составляющая скорости; $\varrho-$ плотность; $\tau-$ продольная координата в фиктивном пространстве.

Общий интеграл уравнения (1) при граничных условиях

$$
\frac{\partial Q u^{2}}{\partial y}(\tau ; \infty)=0, \frac{\partial Q u^{2}}{\partial y}(\tau ;-\infty)=0
$$

Работа выполнена в секторе теплофизики Института термофизики и электрофизики АН Эстонской ССР под руководством доктора технических наук Ю. Иванова. 
может быть получен методом Фурье и выражается для несжимаемой жидкости формулой

$$
\left(\frac{u}{u_{0 m}}\right)^{2}=\frac{1}{2 \sqrt{\pi \tau}} \int_{-\infty}^{\infty} f(v) \exp \left[-\frac{(v-y)^{2}}{4 \tau}\right] d v
$$

Начальное распределение величины $f(v)=\left(\frac{u_{0}}{u_{0 m}}\right)^{2}$ в общем случае может быть задано различными функциями, зависящими от конструктивных особенностей сопел. Для простоты его можно задать полиномом второй степени

$$
f(v)=p v^{2}+q v+1
$$

непрерывным на участке $-1<v<1$ (все линейные размеры отнесены к полуширине струи $b_{0}$ ), а также

$$
f(v)=0\left\{\begin{array}{l}
1<v<\infty \\
-\infty<v<-1
\end{array}\right.
$$

С учетом (4) уравнение (3) запишется в виде

$$
\left(\frac{u}{u_{0 m}}\right)^{2}=\frac{1}{2 \sqrt{\pi \tau}} \int_{-1}^{1}\left(p v^{2}+q v+1\right) \exp \left[-\frac{(v-y)^{2}}{4 \tau}\right] d v
$$

Здесь также все линейные размеры отнесены к полуширине струи $b_{0}$ в соответствующей степени

$$
v=\frac{v_{\text {абс }}}{b_{0}}, \quad \tau=\frac{\tau_{\text {абс }}}{b_{0}^{2}}, \quad x=\frac{x_{\text {абс }}}{b_{0}}
$$

(индекс «абс» означает абсолютные размеры, например мм).

После подстановки (4) в (3) и интегрирования получаем формулу

$$
\begin{gathered}
\left(\frac{u}{u_{0 m}}\right)^{2}=\frac{1}{2}\left(p y^{2}+q y+1+2 p \tau\right)\left(\operatorname{erf} \frac{1-y}{2 \sqrt{\tau}}+\operatorname{erf} \frac{1+y}{2 \sqrt{\tau}}\right)- \\
-\sqrt{\frac{\tau}{\pi}}(p+q+p y) \exp \left[-\frac{(1-y)^{2}}{4 \tau}\right]-\sqrt{\frac{\tau}{\pi}}(p-q-p y) \exp \left[-\frac{(1+y)^{2}}{4 \tau}\right]
\end{gathered}
$$

(здесь $\operatorname{erf} z=\int_{0}^{z} e^{-t^{2}} d t-$ интеграл вероятности).

Для равномерного начального профиля, когда $p=q=0$, формула (6) превращается в хорошо известную формулу

$$
\left(\frac{u}{u_{v m}}\right)^{2}=\frac{1}{2}\left(\operatorname{erf} \frac{1-u}{2 \sqrt{\tau}}+\operatorname{erf} \frac{1+y}{2 \sqrt{\tau}}\right) .
$$

Чтобы иметь возможность воспользоваться формулой (6), нужно определить функцию $\tau(x)$. По данным Райхардта и опытам Б. Устименко [3], для затопленной струи с равномерным начальным профилем 
$\tau(x)=k x^{2}$, в частности в работе [3] $\tau(x)=0,0022 x^{2}$ (где $k-$ коэффициент пропорциональности, характеризующий интенсивность поперечного переноса количества движения). Остается установить, влияет ли форма начального профиля на функцию $\tau(x)$. Непосредственно из опыта это установить трудно, так как меры по изменению формы начального профиля могут изменить и коэффициент $k$, и нам не удастся выделить явление в чистом виде. Поэтому мы обратились к опытам по взаимодействию двух струй, вытекающих из сопел с параллельными осями. Такую систему можно рассматривать как плоскопараллельную струю со ступенчатым начальным профилем.

Опыты показали, что профили в поперечном сечении удовлетворительно описываются формулой (3) при подстановке соответствующих начальных условий. Что же касается функции деформации $\tau(x)$, то при нулевом расстоянии между соплами она практически не зависит от соотношения скоростей (рис. 1). Эти данные позволяют предположить, что функция $\tau(x)$ не зависит от формы начального профиля, заданного и другими функциями.

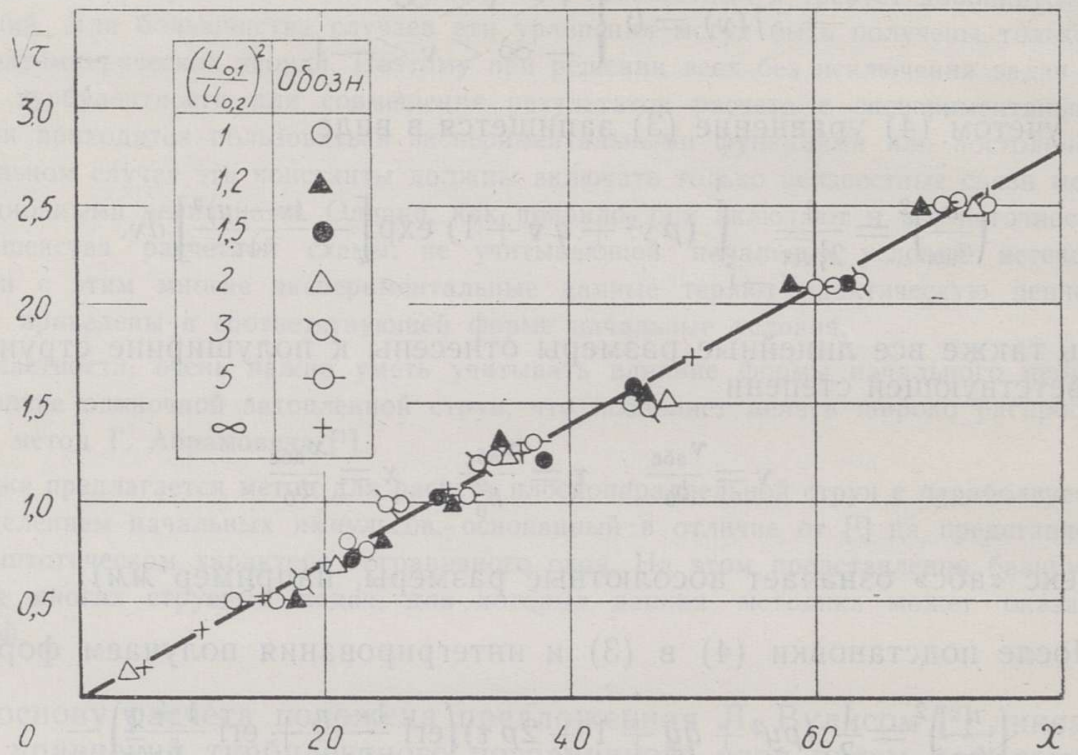

Рис. 1. Функции деформации координат для струи со ступенчатым начальным профилем.

Сравнение расчетов по формуле (6) с экспериментом (начальное распределение полных напоров исследуемой струи удовлетворительно описывалось формулой $\left.\left(\frac{u_{0}}{u_{0 m}}\right)=-0,4 y^{2}-0,1 y+1\right)$ показало, что формула (6) лучше, чем (7) описывает профили в поперечном сечении струи, особенно при больших значениях $y$ (рис. 2). Значение функции $\tau(x)=0,0022 x^{2}$, полученное при совмещении расчетных и экспериментальных значений в точке $y=0$, совпадает с данными [3] и нашими опытами для струи с равномерным начальным профилем. Это еще раз подтверждает принятое предположение, что функция $\tau(x)$ не зависит от формы начального профиля.

Так как расчеты по уравнению (6) приводят к довольно громоздким вычислениям, для приближенных расчетов может быть рекомендована формула $(7)$, где $\tau(x)$ заменена $\tau_{1}(x)$. 

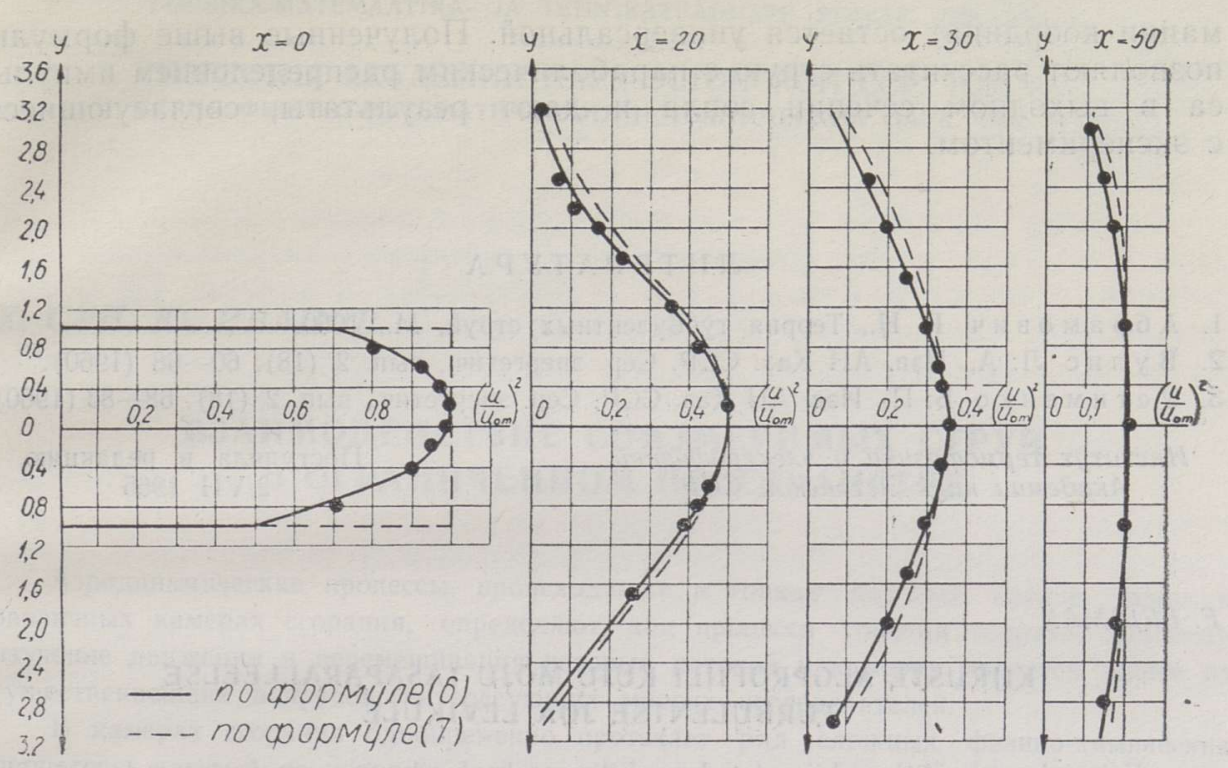

Рис. 2. Развитие струи с неравномерным начальным профилем.

Функция $\tau_{1}(x)$ определится из следующих соображений. В случае. равномерного начального профиля затухание импульса на оси струи получится из формулы (7) при $y=0$

$$
\left(\frac{u_{m}}{u_{0 m}}\right)_{\text {рав }}^{2}=\operatorname{erf} \frac{1}{2 \sqrt{\tau}} .
$$

Аналогично из формулы (6) для параболического профиля

$$
\left(\frac{u_{m}}{u_{0 m}}\right)^{2}=(1+2 a \tau) \operatorname{erf} \frac{1}{2 \sqrt{\tau}}-2 p \sqrt{\frac{\tau}{\pi}} \exp \left[-\frac{1}{4 \tau}\right] .
$$

Разделив (6a) на (7a) и разложив соответствующие функции в степенные ряды, получим

$$
\alpha=\frac{\left(\frac{u_{m}}{u_{0 m}}\right)^{2}}{\left(\frac{u_{m}}{u_{0 m}}\right)_{\mathrm{pas}}^{2}}=1+0,33 p\left(\mathrm{I}-\frac{0,07}{\tau}\right)
$$

и, наконец,

$$
\left(\frac{u_{m}}{u_{0 m}}\right)^{2}=\operatorname{erf} \frac{1}{2 \sqrt{\tau_{1}}}=\alpha \operatorname{erf} \frac{1}{2 \sqrt{\tau}}=\left[1+0,33 p\left(1-\frac{0,0 \vec{\imath}}{\tau}\right)\right] \operatorname{er}\left[\frac{1}{2 \sqrt{\tau}},\right.
$$

¿а так как $\tau(x)=0,0022 x^{2}$,

TO

$$
\operatorname{erf} \frac{1}{2 \sqrt{\tau_{1}}}=\left[1+0,33 p\left(1-\frac{32}{x^{2}}\right)\right] \operatorname{erf} \frac{1}{0,09 x} \text {. }
$$

Формула (9) может быть использована только для $x>15$.

Таким образом, форма начального профиля скоростных напоров: оказывает влияние как на форму профиля в поперечном сечении струи, так и на затухание осевого импульса, в то время как функция дефор- 
мации координат остается универсальной. Полученные выше формулы позволяют рассчитать струю с параболическим распределением импульса в выходном сечении сопла и дают результаты, согласующиеся с экспериментом.

\section{Л И Т Е Р А У P A}

1. Абрамович Г. Н., Теория турбулентных струй, М., 1960.

2. В ули с Л. А., Изв. АН Каз. ССР. Сер. энергетич., вып. 2 (18), 60-68 (1960).

3. У с т и менко Б. П., Изв. АН Каз. ССР. Сер. энергетич., вып. 2 (18), 68 - 83 (1960).
Институт термофизики и электрофизики Академии наук Эстонской ССР

\section{Поступила в редакцию} 2/VII 1965

\section{F. FRISMAN}

\section{KIIRUSTE ALGPROFIILI KUJU MÕJU TASAPARALLEELSE TURBULENTSE JOA LEVIKULE}

Esitatakse ebaühtlase kiiruste algprofiiliga vaba lapiku joa arvutusmeetod, mis põhineb ecldusel, et piirkiht on asümptootilise iseloomuga.

Algvõrrandiks on lineariseeritud liikumisvõrrand, taandatuna soojusjuhtivuse võrranditüübile fiktiivses ruumis. Nagu näitavad katsed, ei olene üleminekufunktsioon fiktiivselt ruumilt reaalsele kiiruste algprofiili kujust.

\section{F. FRISHMAN}

\section{INFLUENCE OF THE INITIAL VELOCITY PROFILE ON THE DEVELOPMENT OF A TWO-DIMENSIONAL FREE TURBULENT JET}

A method of calculation of a free two-dimensional jet with non-uniform initial velocity profile is proposed, which is based on the conception of the asymptotic character of the boundary layer.

The basic equation is a linearized equation of motion transformed to the typical equation of heat conduction in fictitious space.

As the experiment implicates, the function of transformation from fictitious to real space does not depend on the form of the initial velocity profile. 